\title{
The distribution of dark matter around galaxies: Mean surface density profile and outer structure
}

\section{Shogo Masaki*}

Department of physics, Nagoya University

E-mail: masaki@a.phys.nagoya-u.ac.jp

\begin{abstract}
We study the matter distribution in and around dark halos in the standard $\Lambda$ cold dark matter $(\Lambda \mathrm{CDM})$ universe. We use the outputs of high resolution $N$-body simulations to compute the mean surface density profile around galactic size dark matter halos. The result is compared with the observed surface density profile from the lensing magnification measurement of quasars in Sloan Digital Sky Survey (SDSS) recently reported by Ménard et al. (2010). We show that the $\Lambda \mathrm{CDM}$ simulations reproduce the observed surface density profile remarkably well over a wide range of radial distance of $10-10^{4} h^{-1} \mathrm{kpc}$. The mean surface density profile at small separation lengths is consistent with the theoretical prediction that dark halos have a universal NavarroFrenk-White density profile. The observed single power-law of $\Sigma \propto R^{-0.8}$ over the distance range is explained by a simple analytic model based on a halo approach. We also study the distribution of dark matter in the outer part of the halos by measuring the mass enclosed within $\alpha R_{\text {virial }}$, as a function of $\alpha$. Our simulations suggest that more than a half of total matter is contained in about $10 \%$ volume associated with galactic halos. Assuming the SDSS galaxies are hosted by dark halos with mass $2 \times 10^{12}-10^{14} M_{\odot}$, the total mass around the halos amounts to 30 percent of the total mass in the local universe.
\end{abstract}

Identification of Dark Matter 2010

July 26 - 302010

University of Montpellier 2, Montpellier, France

\footnotetext{
* Speaker.
} 


\section{MEAN SURFACE DENSITY PROFILE AROUND GALAXIES FROM WEAK LENSING}

The surface density profile is the projection of the three dimensional density distribution along the line-of-sight,

$$
\Sigma(R)=\int \rho\left(r=\sqrt{R^{2}+\chi^{2}}\right) d \chi
$$

where $r$ is three dimensional distance, $\chi$ is the line-of-sight, and $R$ is two dimensional distance in the lens plane. The mass surface density of lens objects can be probed through the magnification $\mu$ [1] or the tangential shear $\gamma_{t}[2]$. In weak lensing regime, they can be related to the surface density via the following equations:

$$
\begin{aligned}
& \mu(R)=1+2 \kappa(R)=1+2 \frac{\Sigma(R)}{\Sigma_{\mathrm{cr}}}, \\
& \gamma_{\mathrm{t}}(R)=\frac{\bar{\Sigma}(<R)-\Sigma(R)}{\Sigma_{\mathrm{cr}}}=\frac{\Delta \Sigma(R)}{\Sigma_{\mathrm{cr}}},
\end{aligned}
$$

where $\bar{\Sigma}(<R)$ is the average of $\Sigma$ within $R, \kappa$ is the convergence and $\Sigma_{\mathrm{cr}} \equiv \frac{c^{2}}{4 \pi G} \frac{D_{\mathrm{s}}}{D_{\mathrm{ds}} D_{\mathrm{d}}}$ is the critical surface density. Using these relation, one can derive the mean surface density profile of sample galaxies, groups or clusters from the observed average shear or magnification.

Ménard et al. ([1], hereafter M10) recently measured the mean surface density profile of SDSS galaxies with the mean redshift $\langle z\rangle=0.36$. They calculated the cross correlation between the number density fluctuation of foreground galaxies and the flux magnification fluctuation of background quasars. The derived $\Sigma(R)$ of M10 has two interesting features. Firstly, it is well described by a power law $\Sigma \propto R^{-0.8}$. One may naively expect that, if the galactic halos have Navarro-Frenk-White (NFW) density profiles [3], the surface density decreases as $\propto R^{-2}$ at large radii. The observed power-law does not appear to agree with this expectation. It is clearly important to study if the observed surface density profile is consistent with the matter distribution in the standard $\Lambda$ cold dark matter $(\Lambda \mathrm{CDM})$ model.

The second interesting feature is that the power law form of the mean surface density profile continues from $\sim 10 h^{-1} \mathrm{kpc}$ to $\sim 10 h^{-1} \mathrm{Mpc}$. This implies that the galaxy have a very extended dark matter halo, well beyond the typical virial radius of galaxies of about $100 \mathrm{~h}^{-1} \mathrm{kpc}$.

In this work, we study the matter distribution in and around galactic halos for the standard cosmological model. We use high-resolution $N$-body simulations and analytic models to predict surface density profiles. We compare the predictions with the observed surface density profile obtained from the magnification measurement. Also, we measure the amount of dark matter in outer region of halos.

\section{METHOD}

\subsection{Cosmological $N$-body Simulation}

We use the parallel $N$-body solver Gadget-2 [4] 5] in its Tree-PM mode. The cosmological parameters we adopted are, with the standard notations, $\Omega_{m}=0.258, \Omega_{b}=0.0441, \Omega_{\Lambda}=0.742$, $h=0.719$ in units of $100 \mathrm{~km} \mathrm{~s}^{-1} \mathrm{Mpc}^{-1}, n_{s}=0.963, \sigma_{8}=0.796$. These parameters are consistent 
with the WMAP five-year results [6. We employ $1024^{3}$ particles in a box of comoving $200 h^{-1} \mathrm{Mpc}$ on a side. The mass of a dark matter particle is then $5.34 \times 10^{8} h^{-1} M_{\odot}$. Hence we are able to locate dark halos with mass a few times $10^{10} h^{-1} M_{\odot}$ robustly. We set the gravitational softening parameter $\varepsilon=10 h^{-1} \mathrm{kpc}$.

We set the initial redshift $z_{i}=50$ and generate the initial condition using the second-order Lagrangian perturbation theory (2LPT) [7 rather than the standard Zel'dovich approximation (ZA) [8]. 2LPT is more accurate to generate initial conditions and has an advantage to allow us to set the initial redshift more later than ZA. The initial matter power spectrum at $z=z_{i}$ is computed by $C A M B$ [9] for the adopted cosmological parameters.

\subsection{Halo Identification}

Halo identification is done in a two-step manner. First, we select candidate objects using the friends-of-friends (FOF) algorithm [10]. We set the linking parameter $b=0.2$.

We apply the spherical over density algorithm to the located FOF groups that contains 200 particles at least. To each FOF group, we assign a mass such that the enclosed mass within the virial radius is $\Delta \times \rho_{\text {crit }}(z)$. Based on the spherical collapse model, $\Delta$ is set to 200 . We set the minimum number of particles in a halo to 100 and have identified 229,805 halos with the masses greater than $5.34 \times 10^{10} h^{-1} M_{\odot}$ at $z=0.36$.

\section{SURFACE DENSITY PROFILE AROUND HALOS}

\subsection{Mean Surface Density Profile Around Halos}

The mean surface density profile around the halos is computed in the following manner. We shift the simulation volume to be centered on a halo. We then project the mass (simulation particles) within comoving $100 h^{-1} \mathrm{Mpc}$ thickness and compute the surface density around the center as a function of projected distance. Finally, we calculate the average of the profiles of all the selected halos, to obtain the mean surface density profile.

Figure 1 compares our simulation result with the M10 data. The horizontal axis is the physical distance at $z=0.36$ from the center of halo. The simulation result agrees with the observational result rather well, both in the amplitude and in the overall shape. The mass range of the central halos used in the ensemble averaging is from $2 \times 10^{12}$ to $1 \times 10^{14} h^{-1} M_{\odot}$. This sample consists of 9,970 halos. The red dashed line shows the contribution from the central halo truncated at its virial radius, the so-called one-halo term. Clearly, the mean surface density at $R \lesssim 100 h^{-1} \mathrm{kpc}$ is dominated by the one-halo term.

\subsection{The Effect of the Mass Range}

We now examine how the choice of the halo mass range affects the mean surface density. In the previous sections, the mass range of the central halos used to the ensemble averaging is fixed to $2 \times 10^{12}<M /\left(h^{-1} M_{\odot}\right)<1 \times 10^{14}$. Here, we compute the mean surface density profiles by varying the low mass threshold. The upper limit is fixed to $1 \times 10^{14} h^{-1} M_{\odot}$ and the five adopted lower limits $M_{\text {lower }}\left[h^{-1} M_{\odot}\right]$ are as follows : $5 \times 10^{11}, 2 \times 10^{12}, 5 \times 10^{12}, 2 \times 10^{13}, 5 \times 10^{13}$. Note 


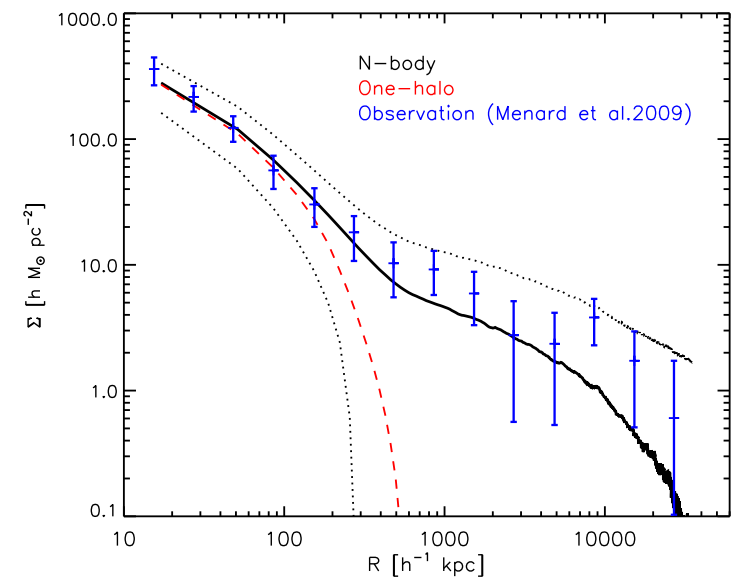

Figure 1: We compare the mean surface density profile in our $\Lambda \mathrm{CDM}$ simulation (black solid line, with $1 \sigma$ deviations denoted by the dotted lines) with the M10's observation (blue symbols with error bars). The mass range of halo sample used in the ensemble averaging is $2 \times 10^{12}-1 \times 10^{14} h^{-1} M_{\odot}$. The horizontal axis is the physical distance at $z=0.36$ from the center of halo. The red dashed line shows the contribution from the so-called one-halo term.

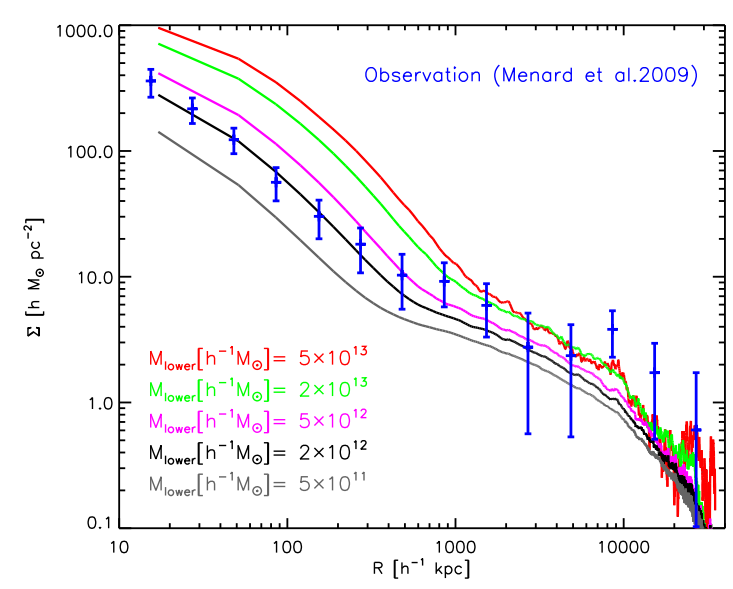

Figure 2: Mean surface density profiles around halos for cases with five different lower mass limits. The amplitude at $R>1 h^{-1} \mathrm{Mpc}$ only weakly depends on $M_{\text {lower }}$ but rather strongly at $R<1 h^{-1} \mathrm{Mpc}$. The former can be explained by using halo - matter two point correlation function while the latter reflects the effect of halo mass function on the ensemble averaging (see text).

that the choice of the upper mass is unimportant because of the steep mass function at the large mass.

The mean surface density profiles for the five cases are shown in Figure 2 . At $R \gtrsim 10 h^{-1} \mathrm{Mpc}$, the difference between the mean profiles is relatively small. We assume that galaxies locate at the center of halos. Then that large-scale behaviour can be understood by using the two-point crosscorrelation function between halo and matter, $\xi_{\mathrm{hm}}$. The observed mean surface density profile around halos can be related to $\xi_{\mathrm{hm}}$ via

$$
\Sigma(R)=\bar{\rho}_{\mathrm{m}} \int \xi_{\mathrm{hm}}\left(r=\sqrt{R^{2}+\chi^{2}}\right) d \chi,
$$

where $\bar{\rho}_{\mathrm{m}}$ is the mean matter density. Hence, $\Sigma$ reflects the properties of $\xi_{\mathrm{hm}}$ directly. The similar dependence of $\xi_{\mathrm{hm}}$ on the halo mass range are found in [11].

At $R<1 h^{-1} \mathrm{Mpc}$, the amplitude of the mean surface density has a strong dependence on $M_{\text {lower }}$. Because among the samples used to calculate the mean surface density, the lower mass halos are dominant in number.

Since the one-halo term dominates the amplitude of the surface density at small length scales, the choice of $M_{\text {lower }}$ significantly affects the mean density. This in turn suggest that, the characteristic mass of the galaxies in observational data can be inferred from the mean surface density.

\section{AMOUNT OF DARK MATTER ASSOCIATING HALOS}

In this section, we use our simulation outputs at $z=0.36$ to study the amount of dark matter 
associated with halos. We measure the enclosed mass within $\alpha R_{\text {vir }}$ around halos. We progressively increase the value of $\alpha$ from 1 to 30 and calculated the total enclosed mass around halos. For clarify, the total mass in $\alpha R_{\text {vir }}$ is converted to the density parameter :

$$
\Omega_{\text {halo }}(\alpha) \equiv \frac{\sum_{i} M_{\alpha-\text { halo }, i} / L_{\text {box }}^{3}}{\rho_{\text {crit }}}
$$

where $L_{\mathrm{box}}$ is the box size of our simulation and $M_{\alpha-\text { halo, } i}$ is the mass of $i$ th halo. Similarly, the total volume occupied is expressed as

$$
V_{\text {halo }}(\alpha)=\sum_{i} \frac{4 \pi}{3}\left(\alpha R_{\mathrm{vir}, i}\right)^{3}
$$

where $R_{\mathrm{vir}, i}$ is the virial radius of $i$ th halo. Strictly speaking, these expressions are not exact because there are some overlap region between two or more halos. We simply avoid double counting in mass and in volume when we calculate $\Omega(\alpha)$ and $V(\alpha)$.
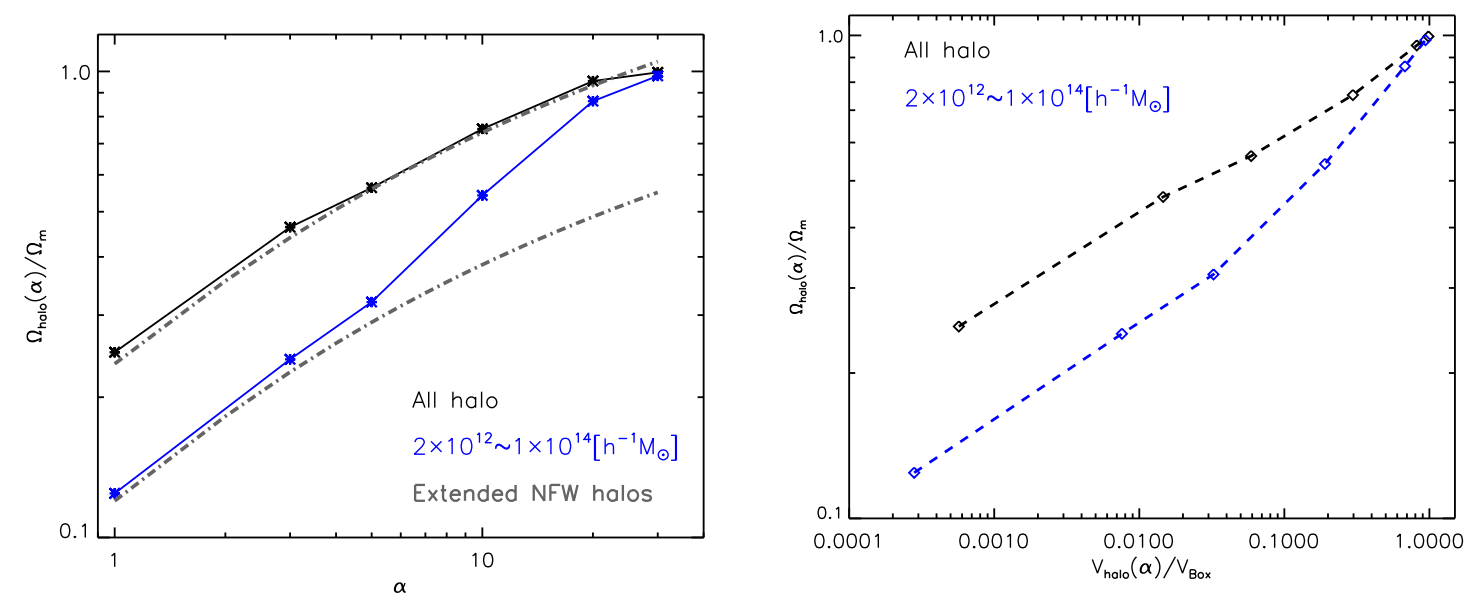

Figure 3: The mass around halos within $\alpha R_{\mathrm{vir}}$. We Figure 4: We plot $\Omega_{\mathrm{halo}} / \Omega_{\mathrm{m}}$ as a function of show the total mass density $\Omega_{\text {halo }}$ scaled by $\Omega_{\mathrm{m}}$ as a $V_{\text {halo }}$ scaled by the whole volume of simulation box. function of the scale parameter $\alpha$. We use all the halos There are six data points corresponding to $\alpha=$ located in our simulation. The data points are from $1,3,5,10,20$ and 30 from left. More than a half of our simulation and the grey dotted-dashed line shows mass is contained within about $10 \%$ of the volume a simple model prediction assuming NFW halos. $\quad(\alpha=5$ in case of all halo).

Figure 3 shows the dependence of $\Omega_{\text {halo }}(\alpha)$ on $\alpha$. The upper solid line with symbols is the result for all halos identified in our simulation, and the lower solid line with symbols is for the samle of halos with masses $2 \times 10^{12} \sim 1 \times 10^{14} h^{-1} M_{\odot}$, respectively. These are direct measurements from our simulations, whereas the grey dotted-dashed lines are simple model predictions assuming halos have infinitely extended NFW density profiles. Clearly, the extended NFW model agrees with $N$ body result well up to $\alpha \simeq 20$ in the case that all halos are used. From this, it is expected that halos have no clear edge. However for the "galactic halo" sample, the model reproduces the simulation result to $\alpha \simeq 5$ but significantly deviates for larger $\alpha$. In this case, the contributions from the 
neighouring halos (not included in the sample) to $\Omega_{\text {halo }}$ obtained from $N$-body outputs directly is expected to increase at large $\alpha$ while the extended NFW model does not account the contribution. The discrepancy between the above two cases occurs due to this.

Figure 4 shows $\Omega_{\text {halo }}$ versus $V_{\text {halo }}$. From this figure, we easily infer that there are more than a half of mass within about $10 \%$ of the volume that are associated with halos $(\alpha=5)$. Assuming the SDSS galaxies used in M10 are hosted by halos with mass $2 \times 10^{12} \sim 1 \times 10^{14} h^{-1} M_{\odot}$ (see Section 3.1 ), about $30 \%$ of the total mass is enclosed in them and the corresponding volume occupied is about $5 \%$.

\section{SUMMARY}

We showed that $\Lambda \mathrm{CDM} N$-body simulation can reproduce the surface density profile around galaxies observed by Ménard et al. [1] very well. It is also shown that the amplitude of the inner surface density profile can be an estimator of the typical halo mass of the sample galaxies, and that the observed surface density profile can be described well by a halo model.

We also investigated the distribution and amount of dark matter in the outer part of the halos. Our simulations suggest that the total mass around SDSS galaxies' host halos amounts to $30 \%$ of the total mass in the local universe. It is also implied that halos do not have a clear edge but a rather extended envelope.

\section{References}

[1] Ménard, B., Scranton, R., Fukugita, M., \& Richards, G. 2010, MNRAS, 405, 1025

[2] Sheldon, E. S., et al. 2004, AJ, 127, 2544

[3] Navarro, J. F., Frenk, C. S., \& White, S. D. M. 1997, ApJ, 490, 493

[4] Springel, V., Yoshida, N., \& White, S. D. M. 2001, New Astronomy, 6, 79

[5] Springel, V. 2005, MNRAS, 364, 1105

[6] Komatsu, E., et al. 2009, ApJS, 180, 330

[7] Scoccimarro, R. 1998, MNRAS, 299, 1097

[8] Zel'Dovich, Y. B. 1970, A\&A, 5, 84

[9] Lewis, A., Challinor, A., \& Lasenby, A. 2000, ApJ, 538, 473

[10] Davis, M., Efstathiou, G., Frenk, C. S., \& White, S. D. M. 1985, ApJ, 292, 371

[11] Hayashi, E., \& White, S. D. M. 2008, MNRAS, 388, 2 